See the related article beginning on page 1563.

\title{
CD44 - a sticky target for asthma
}

\author{
Marc E. Rothenberg
}

Division of Allergy and Immunology, Department of Pediatrics, Cincinnati Children's Hospital Medical Center, Cincinnati, Ohio, USA

J. Clin. Invest. 111:1460-1462 (2003). doi:10.1172/JCI200318392.

One of the paradoxes of modern medicine is the rapidly growing incidence of immune-based diseases over the last half of the century (1). Despite enormous advances in our understanding of the immune system, and our ability to manipulate immunity in experimental animals and man, we have not been able to curtail these diseases. In fact, it is becoming increasingly evident that immune hypersensitivity responses are central to the pathogenesis of many of the most common diseases of the 21 st century including atherosclerosis, diabetes, obesity, and arthritis (2). Included in this epidemic are atopy-associated disorders (such as asthma, eczema, allergic rhinoconjunctivitis, and food allergies), which have skyrocketed during this time frame (about a threefold increase in prevalence). Currently, in the United States, nearly $30 \%$ of the population suffers from allergies with $5-10 \%$ suffering from asthma, a chronic inflammatory pulmonary disorder that is a chief diagnosis responsible for pediatric hospital admission, work and school absenteeism, and annual health care expenditures exceeding 12 billion dollars. While genetic factors certainly contribute to the pathogenesis of these diseases, there is emerging evidence that their rising incidence is

\footnotetext{
Address correspondence to: Marc E. Rothenberg, Division of Allergy and Immunology, Department of Pediatrics, Cincinnati Children's Hospital Medical Center, 3333 Burnet Avenue, ML7028, Cincinnati, Ohio 45229, USA.

Phone: (513) 636-7177; Fax: (513) 636-3310;

E-mail: Rothenberg@chmcc.org.

Conflict of interest: The author has declared

that no conflict of interest exists.

Nonstandard abbreviations used:

macrophage chemoattractant protein (MCP); thymus- and activation-regulated chemokine (TARC); leukotriene (LT); hyaluranon (HA).
}

related to changes in western lifestyle. For example, with increased time spent indoors (and its associated increased exposure to ubiquitous common antigens (derived from fungi, dust mites, and home pets), less exposure to rural outdoor adjuvants (e.g., endotoxin from farm animals), increased use of antibiotics and vaccinations (and the associated changes in infections and endogenous flora), there is emerging evidence that the immune system has become "delinquent", capable of eliciting the adverse responses associated with these disorders (3). Indeed, alterations in regulatory $T$ cells (and TGF- $\beta$ production) have been associated with these changes (4).

Asthma is a disease defined by reversible airflow obstruction, and increased lung responsiveness to a variety of antigen-specific (allergens) and -nonspecific (e.g., cold air, cigarette smoke) triggers, strongly associated with atopy (IgE production) (5). Histologically, the asthmatic lung is characterized not only by eosinophilrich inflammation, but also by a variety of chronic changes that induce lung remodeling (including mucus production, smooth muscle hyperplasia, and deposition of extracellular matrix components) (6). There is strong evidence that $\mathrm{CD}^{+} \mathrm{T}$ cells, especially of the Th2 phenotype, induce many of the features of asthma through the secretion of an array of cytokines (e.g., IL-4, IL-5, IL-9, IL-13, and IL-25) that activate inflammatory and residential effector pathways both directly and indirectly (7). Notably, IL-4 and IL-13 directly trigger airway cells to undergo proasthmatic changes including production of mucus, expression of a variety of specific adhesion molecules (e.g., ICAM-1 and VCAM-1), and production of a select sub-group of chemokines (eotaxins, macrophage chemoattractant proteins [MCPs]), and thymusand activation-regulated chemokine [TARC]) that attract and activate allergic inflammatory cells (e.g., eosinophils, macrophages, and Th2 cells) (8). The partial clinical effectiveness of glucocorticoids (agents that inhibit the production of Th2 cytokines, eosinophils, and T cells), cysteinylleukotriene (LT) inhibitors, and anti-IgE therapeutics indicates the critical interplay of multiple cells, cytokines, and mediators in disease pathogenesis.

Leukocyte migration into the allergic lung has been eloquently demonstrated to be dependent upon the coordinate interplay between selectins, adhesion molecules, and chemoattractant molecules (including chemokines and LTs). In particular, reversible interactions between Th2 cells and eosinophils with vascular endothelial cells are primarily mediated by selectins; whereas firm adhesion and cellular transmigration is primarily triggered by integrins of the $\beta 1$ and $\beta 2$ families. The $\alpha 4 \beta 1$ integrin (also known as very late antigen-4) has been shown to be particularly important since it is co-expressed by multiple leukocytes involved in allergic inflammation (including $\mathrm{T}$ cells and eosinophils) and treatment with anti$\alpha 4$ antibodies inhibits many essential features of experimental asthma in animals (9). Classically, adhesion events involved in leukocyte migration into inflammatory sites have focused on the role of soluble chemoattractant mediators; however, components of the extracellular matrix can also strongly influence inflammatory cell recruitment and activation. For example, hyaluronan (HA) - a glycosaminoglycan composed of repeating units of $D$-glucuronate $(1-\beta-3)$ and $\mathrm{N}$-acetyl- $D$-glucosamine $(1-\beta-4)$ - and fibronectin can directly induce eosinophil chemoattraction, survival, and function (10). Although these and other extracellular matrix molecules can bind to a variety of cell surface receptors, CD44 appears to be a particularly important leukocyte receptor for HA and may therefore have a role in the pathogenesis of 


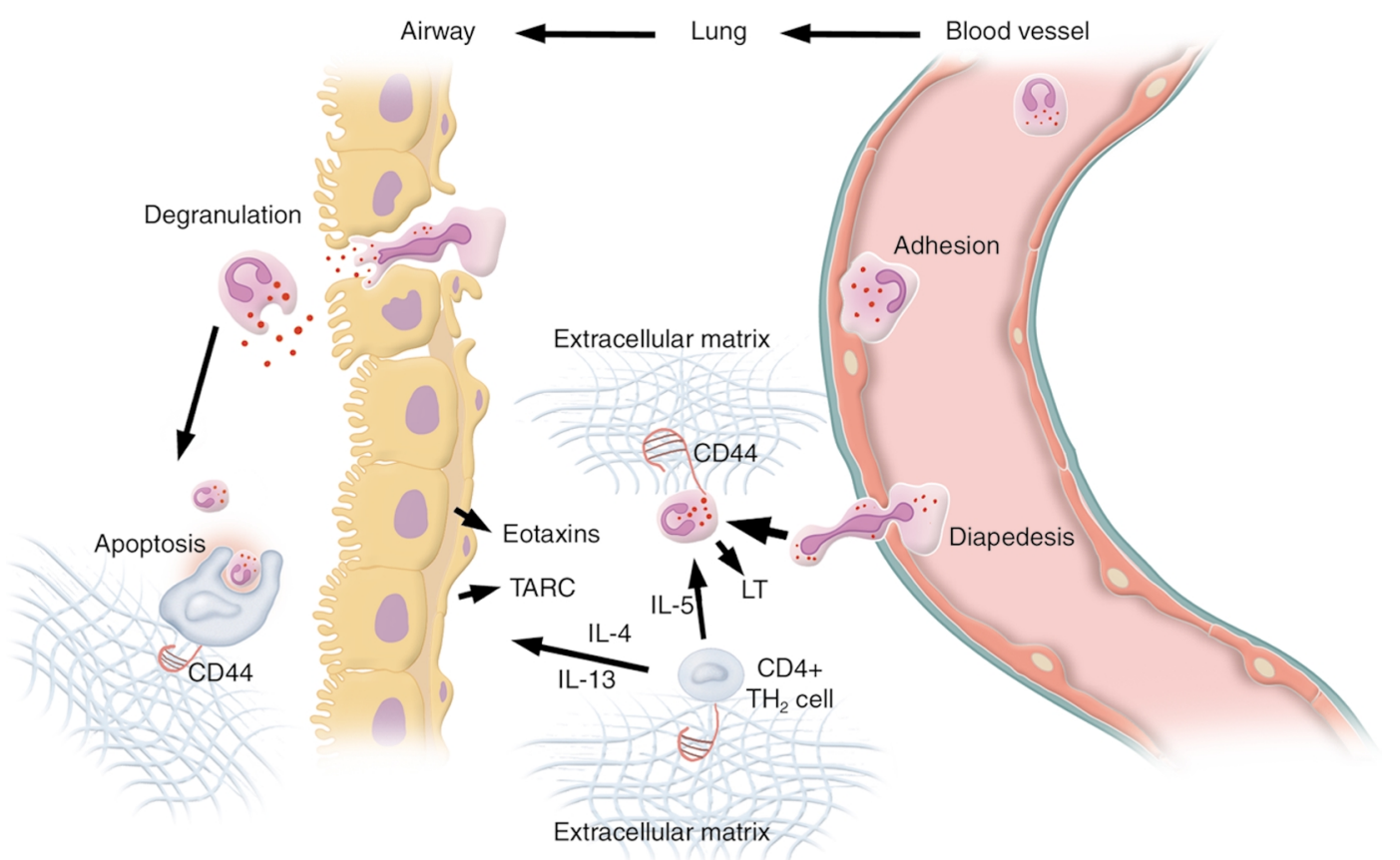

\begin{abstract}
Figure 1
Overview of immunological mechanisms in the development of allergic eosinophilic airway inflammation involving CD44. There are multiple mechanisms by which CD44 may effect the propagation and resolution of allergic airway inflammation. Engagement of CD44 by a variety of ligands (typically extracellular matrix components such as $\mathrm{HA}$ ) on $\mathrm{CD} 4+\mathrm{T}$ cells and eosinophils promotes cellular chemotaxis, activation, and survival. Th2 cell-derived IL-4 and IL-13 potently induce critical chemokines (including TARC and the eotaxins) by several resident cells in the lung (e.g., respiratory epithelium), and induce direct asthma responses (including mucus production and airway hyperreactivity). Eosinophils further exacerbate allergic inflammation by production of potent smooth muscle constrictors (e.g., LTs) and by the cytotoxic effects of their granule proteins on lung cells. CD44 may also have an important role in resolution of allergic airway inflammation by promoting the removal of apoptotic airway cells by macrophage-mediated CD44-dependent phagocytosis and clearance.
\end{abstract}

asthma. CD44 is a class I transmembrane glycoprotein originally described on leukocytes but now known to be expressed on most vertebrate cells (11). The amino-terminal globular domain serves as a docking site for multiple components of the extracellular matrix including HA, collagen, laminin, and fibronectin. The cytoplasmic tail serves as a docking site for numerous intracellular partners including ankyrin, the actin cytoskeleton, and band 4.1 proteins such as the tumor suppressor protein merlin. Accordingly, CD44 is involved in numerous processes involved in health (e.g., organ development, neuronal axon guidance) and disease (tumor genesis, tumor metastasis, and inflammation) (11).

In this issue of the JCI, Katoh et al. report an impressive role for CD44 in orchestrating allergic eosinophilic airway inflammation (experimental asthma) (12). In their study, the investigators disturbed CD44 function with two different monoclonal antibodies and examined the consequences on two distinct models of antigen-induced experimental asthma. Their antibodies included KM201, a CD44 neutralizing reagent, and IM7, an antibody that induced CD44 shedding. Using both approaches and appropriate isotype matched control antibodies, the investigators demonstrated dose-dependent inhibition of multiple aspects of experimental asthma. Notably, leukocyte recruitment (eosinophil and lymphocyte), Th2 (IL-4, and IL-5) but not Th1 cytokine production, Th2-associated chemokine (e.g., eotaxin and TARC) production, LT induction, and enhanced airway hyperreactivity were all remarkably reduced. In addition, levels of HA, which were increased during experimental asthma in control mice were markedly attenuated in the antibody-treated mice, supporting a role for CD44 in HA metabolism (specifically in the breakdown of high mol wt HA to pro-inflammatory low mol wt forms). This may be particularly important because HA-derived oligosaccharides can bind and activate
Toll-like receptor 4 (13). Clearly, the most impressive aspect of the results presented by Katoh et al. is the profound magnitude of the beneficial effects of anti-CD44 treatment.

The results of Katoh et al. (12) are consistent with several studies demonstrating that CD44 is a pro-inflammatory receptor in a variety of murine models. For example, mice with a disruption of the $\mathrm{CD} 44$ gene or mice treated with anti-CD44 are protected from hapten-induced colitis, collageninduced arthritis, delayed-type hypersensitivity responses in the skin, and experimental atherosclerosis (14-16). However, these results strongly contrast with the recent findings reported by Paul Noble and colleagues (17). In the Noble study, $C D 44^{-/-}$mice developed exaggerated lung-inflammatory responses in a bleomycin-induced model of lung injury. Notably, $C D 44^{-/}$ mice had increased levels of lung leukocytes (neutrophils, macrophages, and lymphocytes), chemokines (e.g., MCP-5), and HA. The authors used 
bone marrow reconstitution studies to show that CD44 expression on hematopoietic cells was responsible for the defect, which the authors attributed to impaired alveolar macrophage CD44-mediated clearance of apoptotic cells in the lung. Thus, in the Noble study, CD44 appears to have a protective antiinflammatory role in the lung. At first glance, these results appear to conflict with the results of Katoh et al. However, there are important differences between the two studies that should be considered. First, the role of CD44 in the bleomycin model was only seen after 5 days of lung inflammation. In contrast, Katoh et al. only examined an acute time point ( 24 hours) after allergen challenge. Second, while $C D 44^{-/-}$mice have a ubiquitous deficiency of CD44, the monoclonal antibody approach taken by Katoh et al. did not deplete CD44 expression by alveolar macrophages (and likely other cell populations). This is important because alveolar macrophages are likely to be the critical cell required for resolution of lung inflammation. And third, great caution has to be taken when comparing germline gene-targeting with conditional CD44 neutralization later in development. Prior studies have shown baseline deficiencies (e.g., in myeloid progenitor cells and in $\mathrm{T}$ cell homing and apoptosis) in $\mathrm{CD}_{44^{-/-}}$ mice (11). Additionally, it has been proposed that loss of CD44 function early in development (embryonic) is not tolerated the same way as when it is only absent in adults, as demonstrated by CD44's role in limb development (11).

\section{A unifying mechanism to explain CD44's role in allergic lung inflammation}

Clearly, CD44 has different functions on different cell populations. Most studies have supported an important role for CD44 in promoting leukocyte recruitment, likely mediated by extracellular matrix components triggering changes in the cytoskeletan via CD44dependent signaling events. The findings of Katoh et al. (12) support an essential role for CD44 in allergeninduced Th2 cell and eosinophil recruitment to the lungs (Figure 1). Whether this represents a primary defect in leukocyte recruitment or a secondary defect in the production of chemoattractants by lung parenchymal cells (e.g. airway epithelium) remains uncertain. It is interesting to speculate that CD44 may also serve to resolve allergen-induced inflammation by macrophage-mediated clearance of lumenal cells, many of which eventually undergo apoptosis. Thus, CD44 may have a protective or disease-promoting role on different phenotypic or temporal aspects of asthma. Thus, while antiCD44 appears to be a potentially attractive new target for asthma therapy, further analyses (e.g. kinetics and cell specific conditional gene targeting) are needed before we can better predict the value of this approach.

\section{Acknowledgments}

Marc E. Rothenberg is supported in part by NIH grants R01 AI42242-05, R01 AI45898-03, the Human Frontier Science Program, the International Life Sciences Institute, and the Burroughs Wellcome Fund.
1. Bach, J.F. 2002. The effect of infections on susceptibility to autoimmune and allergic diseases. N. Engl. J. Med. 347:911-920.

2. Nathan, C. 2002. Points of control in inflammation. Nature. 420:846-852.

3. Wills-Karp, M., Santeliz, J., and Karp, C.J. 2001. The germless theory of allergic disease: revisiting the hygiene hypothesis. Nat. Rev. Immunol. 1:69-75.

4. Umetsu, D.T., McIntire, J.J., Akbari, O. Macaubas, C., and DeKruyff, R.H. 2002. Asthma: an epidemic of dysregulated immunity. Nat. Immunol. 3:715-720.

5. Elias, J.A., et al. 2003. New insights into the pathogenesis of asthma. J. Clin. Invest. 111:291-297. doi:10.1172/JCI200317748.

6. Busse, W.W., and Lemanske, R.F., Jr. 2001. Asthma. N. Engl. J. Med. 344:350-362.

7. Ray, A., and Cohn, L. 1999. Th2 cells and GATA-3 in asthma: new insights into the regulation of airway inflammation. J. Clin. Invest. 104:985-993.

8. Zimmermann, N., Hershey, G.K., Foster, P.S., and Rothenberg, M.R. 2003. Chemokines in asthma: cooperative interaction between chemokines and IL-13. J. Allergy Clin. Immunol. 111:227-242.

9. Schleimer, R.P., and Bochner, B.S. 1998. The role of adhesion molecules in allergic inflammation and their suitability as targets of antiallergic therapy. Clin. Exp. Allergy 28:15-23.

10. Ohkawara, Y., et al. 2000. Activation and transforming growth factor-beta production in eosinophils by hyaluronan. Am. J. Respir. Cell Mol. Biol. 23:444-451.

11. Ponta, H., Sherman, L., and Herrlich, P.A. 2003 CD44: from adhesion molecules to signaling regulators. Nat. Rev. Mol. Cell Biol. 4:33-45.

12. Katoh, S., et al. 2003. A role for CD44 in an antigen-induced murine model of pulmonary eosinophilia. J. Clin. Invest. 111:1563-1570. doi:10.1172/JCI200316583.

13. Termeer, C., et al. 2002. Oligosaccharides of hyaluronan activate dendritic cells via toll-like receptor 4. J. Exp. Med. 195:99-111.

14. Camp, R.L., Scheynius, A., Johansson, C., and Pure, E. 1993. CD44 is necessary for optimal contact allergic responses but is not required for normal leukocyte extravasation. J. Exp. Med. 178:497-507.

15. Mikecz, K., Brennan, F.R., Kim, J.H., and Glant, T.T. 1995. Anti-CD44 treatment abrogates tissue oedema and leukocyte infiltration in murine arthritis. Nat. Med. 1:558-563.

16. Wittig, B.M., Johansson, B., Zoller, M., Schwarzler, C., and Gunthert, U. 2000. Abrogation of experimental colitis correlates with increased apoptosis in mice deficient for CD44 variant exon 7 (CD44v7). J. Exp. Med. 191:2053-2064.

17. Teder, P., et al. 2002. Resolution of lung inflammation by CD 44. Science. 296:155-158. 